Supporting Information for:

\title{
Construction of Double-Stranded Metallosupramolecular Polymers with a Controlled Helicity by Combination of Salt Bridges and Metal Coordination
}

\author{
Masato Ikeda, ${ }^{\dagger}$ Yoshie Tanaka, ${ }^{\dagger}$ Takashi Hasegawa, ${ }^{\dagger, \ddagger}$ \\ Yoshio Furusho, ${ }^{*, \dagger}$ and Eiji Yashima ${ }^{* \dagger,+,}$
}

\begin{abstract}
'Yashima Super-structured Helix Project, ERATO, Japan Science and Technology Agency (JST), 101 Creation Core Nagoya, 2266-22 Moriyama-ku, Nagoya 463-0003, Japan, and Department of Molecular Design and Engineering, Graduate School of Engineering, Nagoya University, Chikusa-ku, Nagoya 464-8603, Japan.
\end{abstract}

E-mail: furusho@yp-jst.jp; yashima@apchem.nagoya-u.ac.jp

\section{Table of Contents}

$\begin{array}{ll}\text { 1. Instruments } & \text { S2 }\end{array}$

2. Materials $\quad$ S2

3. Synthetic Procedures for the Duplexes $\mathbf{1}^{R} \cdot \mathbf{2}$ and $\mathbf{1}^{S} \cdot \mathbf{2}$ and Polymers $\mathbf{3}^{R}$ and $\mathbf{3}^{S} \quad$ S3

4. Absorption and CD Spectra of the Duplexes $\mathbf{1}^{R} \cdot \mathbf{2}$ and $\mathbf{1}^{S} \cdot \mathbf{2} \quad S 7$

5. ${ }^{1} \mathrm{H}$ NMR (500 MHz, $25^{\circ} \mathrm{C}$ ) Spectra of $\mathbf{1}^{\boldsymbol{R}}, \mathbf{2}$, and $\mathbf{1}^{\boldsymbol{R}} \cdot \mathbf{2} \quad \mathrm{S} 7$

6. CSI Mass Spectra of the Duplexes $\mathbf{1}^{R} \cdot \mathbf{2}$ and $\mathbf{1}^{S} \cdot \mathbf{2} \quad$ S8

7. DOSY NMR Spectra of $\mathbf{1}^{R} \cdot \mathbf{2}$ and $\mathbf{3}^{R} \quad$ S9

8. DLS Study of the Double Helical Coordination Polymer $\mathbf{3}^{R} \quad$ S9

9. Effect of Feed Ratio $\quad$ S10

10. Effect of AcOH on the CD spectra and Hydrodynamic Dimension of $\mathbf{3}^{S} \quad$ S10

11. Molecular Mechanics Calculation of a Trimer Model of $\mathbf{3}^{R} \quad S 11$

$\begin{array}{ll}\text { 12. AFM Measurements } & \text { S12 }\end{array}$ 
1. Instruments. Melting points were measured using a Yanako MP-500D micro melting point apparatus and were uncorrected. The ${ }^{1} \mathrm{H}$ and ${ }^{13} \mathrm{C}$ NMR spectra were measured using a Varian UNITY INOVA 500AS spectrometer operating at $500 \mathrm{MHz}$ for ${ }^{1} \mathrm{H}$ and $125 \mathrm{MHz}$ for ${ }^{13} \mathrm{C}$ with residual non-deuterated solvents as the internal references. The IR spectra were recorded using a Jasco Fourier Transform IR-680 Plus spectrophotometer. The absorption and circular dichroism (CD) spectra were measured in a 0.01-cm quartz cell unless otherwise noted using a Jasco V-570 spectrophotometer and a Jasco J-820 spectropolarimeter, respectively. High-resolution fast atom bombardment (FAB) mass spectra were measured using a JEOL JMS-700 at the Research Center for Material Science at Nagoya University. The optical rotations were measured in a 5-cm quartz cell equipped with a temperature controller (EYELA NCB-2100) on a Jasco P-1030 polarimeter in $\mathrm{CHCl}_{3}$. The coldspray ionization timeof-flight (CSI-TOF) mass spectra were recorded on a JEOL JMS-T100CS mass spectrometer. The dynamic light scattering (DLS) measurements were performed using an Otsuka Photal DLS-7070YN spectrometer equipped with a 10mW He-Ne laser $(632.8 \mathrm{~nm})$. Molecular modeling and molecular mechanics (MM) calculations were performed on a Windows XP PC with the Materials Studio package (Version 3.0; Accelrys Inc.). The atomic force microscopy (AFM) measurements were performed using a Nanoscope IIIa microscope (Veeco Instruments, Santa Barbara, CA) in air at ambient temperature with standard silicon cantilevers (NCH, NanoWorld, Neuchâtel, Switzerland) in the tapping mode.

2. Materials. All starting materials and solvents were purchased from commercial suppliers and were used without further purification unless otherwise noted. 


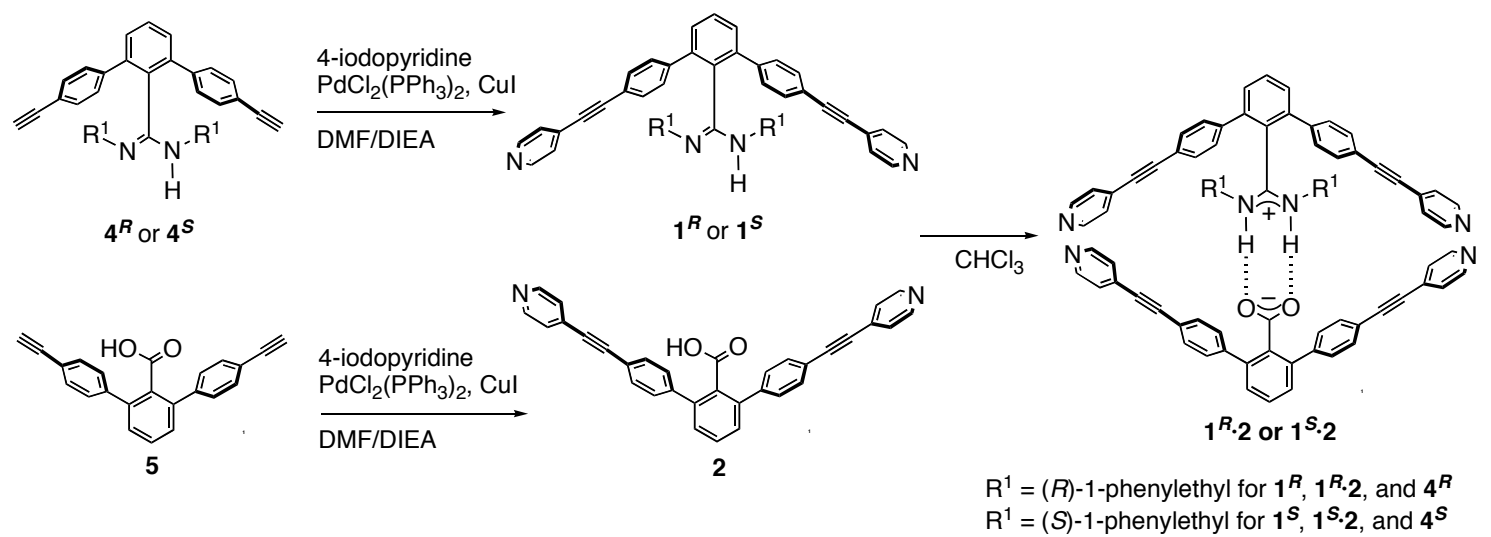

\section{Scheme S1.}

Synthesis of Diacetylenes $4^{R}, 4^{S}$, and 5. $4^{R}, 4^{S}$, and 5 were obtained from the corresponding disilyl compounds by treatment with tetrabutylammonoium fluoride (TBAF) in THF at ambient temperature. ${ }^{1}$

$4^{R}$. Mp: $184.8-185.5{ }^{\circ} \mathrm{C} . \quad{ }^{1} \mathrm{H}$ NMR $\left(500 \mathrm{MHz}, \mathrm{CDCl}_{3}, 4^{R}(30.0 \mathrm{mM}), \mathrm{CH}_{3} \mathrm{CO}_{2} \mathrm{H}(97\right.$ $\left.\mathrm{mM}), 25^{\circ} \mathrm{C}\right): \delta=12.92($ br s, $2 \mathrm{H}, \mathrm{N}-\mathrm{H}), 7.76(\mathrm{t}, J=7.8 \mathrm{~Hz}, 1 \mathrm{H}, \mathrm{ArH}), 7.51$ (d, $J=7.7$ $\mathrm{Hz}, 2 \mathrm{H}, \mathrm{ArH}), 7.32-7.19$ (m, 10H, ArH), 7.02 (d, J=6.9 Hz, 4H, ArH), 6.67 (d, J = 8.5 $\mathrm{Hz}, 4 \mathrm{H}, \mathrm{ArH}), 3.91$ (q, $J=6.8 \mathrm{~Hz}, 2 \mathrm{H}, \mathrm{CHN}), 3.12$ (s, 2H, CCH), 2.09 (s, 10H, $\left.\mathrm{CH}_{3} \mathrm{CO}_{2}\right), 0.70 \mathrm{ppm}\left(\mathrm{d}, J=6.9 \mathrm{~Hz}, 6 \mathrm{H}, \mathrm{CH}_{3}\right)$. Anal. Calcd for $\mathrm{C}_{39} \mathrm{H}_{32} \mathrm{~N}_{2}\left(\mathrm{H}_{2} \mathrm{O}\right)_{0.2}: \mathrm{C}$, 88.00; H, 6.13; N, 5.26. Found: C, 88.12; H, 6.07; N, 5.01.

4. Mp: $184.6-185.5^{\circ} \mathrm{C} . \quad{ }^{1} \mathrm{H}$ NMR $\left(500 \mathrm{MHz}, \mathrm{CDCl}_{3}, 4^{S}\right.$ (20.0 mM), $\mathrm{CH}_{3} \mathrm{CO}_{2} \mathrm{H}(71$ $\mathrm{mM}), 25{ }^{\circ} \mathrm{C}$ ): $\delta=12.99($ br s, $2 \mathrm{H}, \mathrm{N}-\mathrm{H}), 7.76(\mathrm{t}, J=7.8 \mathrm{~Hz}, 1 \mathrm{H}, \mathrm{ArH}), 7.51(\mathrm{~d}, J=7.7$ $\mathrm{Hz}, 2 \mathrm{H}, \mathrm{ArH}), 7.31-7.20$ (m, 10H, ArH), 7.02 (d, J=6.9 Hz, 4H, ArH), 6.67 (d, J = 8.3 $\mathrm{Hz}, 4 \mathrm{H}, \mathrm{ArH}), 3.91$ (q, $J=6.8 \mathrm{~Hz}, 2 \mathrm{H}, \mathrm{CHN}), 3.12$ (s, 2H, CCH), 2.09 (s, 10H, $\left.\mathrm{CH}_{3} \mathrm{CO}_{2}\right), 0.70 \mathrm{ppm}\left(\mathrm{d}, J=6.9 \mathrm{~Hz}, 6 \mathrm{H}, \mathrm{CH}_{3}\right)$. Anal. Calcd for $\mathrm{C}_{39} \mathrm{H}_{32} \mathrm{~N}_{2}: \mathrm{C}, 88.60 ; \mathrm{H}$, 6.10; N, 5.30. Found: C, 88.35; H, 6.19; N, 5.12.

5. Mp: $179.1-179.8{ }^{\circ} \mathrm{C} . \quad{ }^{1} \mathrm{H}$ NMR $\left(500 \mathrm{MHz}, \mathrm{CDCl}_{3}, 5.2 \mathrm{mM}, 2{ }^{\circ} \mathrm{C}\right): \delta=7.56-7.50$ (m, 5H, ArH), 7.38-7.33 (m, 6H, ArH), 3.15 ppm (s, 2H, CCH). ${ }^{13} \mathrm{C}$ NMR (125 MHz, 
$\left.\mathrm{CDCl}_{3}, 42.7 \mathrm{mM}, 25^{\circ} \mathrm{C}\right): \delta=173.01,140.63,139.56,132.13,131.67,129.70,129.12$, 128.45, 121.64, 83.31, 78.14 ppm. FT-IR (KBr): $v=3630(\mathrm{O}-\mathrm{H}), 3294(\mathrm{CC}-\mathrm{H}), 2107$ $(\mathrm{C} \equiv \mathrm{C}), 1698 \mathrm{~cm}^{-1}$. Anal. Calcd for $\mathrm{C}_{39} \mathrm{H}_{32} \mathrm{~N}_{2}\left(\mathrm{CHCl}_{3}\right)_{0.17}: \mathrm{C}, 81.22 ; \mathrm{H}, 4.17$. Found: $\mathrm{C}$, 81.19; H, 3.99.

Synthesis of $\mathbf{1}^{R}$. A solution of compound $4^{R}(150 \mathrm{mg}, 0.28 \mathrm{mmol})$, 4-iodopyridine (257 mg, 4 eq.), $\mathrm{CuI}$ (4.0 mg, $7.5 \mathrm{~mol} \%$ ), $\mathrm{Pd}\left(\mathrm{PPh}_{3}\right)_{2} \mathrm{Cl}_{2}$ (14 mg, $7.5 \mathrm{~mol} \%$ ), and dry DMF ( $5 \mathrm{~mL}$ ) was degassed three times by the freeze pump and thaw (argon) method. To this solution was added dry diisopropylethylamine (DIEA) $(0.5 \mathrm{~mL})$ via syringe and the reaction mixture was stirred overnight at room temperature under an argon atmosphere. The solvent was evaporated to dryness. The residue was purified by flash column chromatography (Fuji-silysia $\mathrm{NH}-\mathrm{SiO}_{2}, \mathrm{CHCl}_{3}$ ) and size exclusion column chromatography (Biobeads SX-1, $\mathrm{CHCl}_{3}$ ) to afford compound $\mathbf{1}^{R}$ in $94 \%$ yield (180 $\mathrm{mg}$ ) as a pale yellow solid. Mp: $111.5-112.6{ }^{\circ} \mathrm{C} . \quad[\alpha]_{\mathrm{D}}{ }^{20}-135\left(c=0.1\right.$ in $\left.\mathrm{CHCl}_{3}\right) . \quad{ }^{1} \mathrm{H}$ $\operatorname{NMR}\left(500 \mathrm{MHz}, \mathrm{CDCl}_{3}, 30{ }^{\circ} \mathrm{C}, \mathbf{1}^{R}(3.0 \mathrm{mM}), \mathrm{CH}_{3} \mathrm{CO}_{2} \mathrm{H}(18 \mathrm{mM})\right): \delta=13.25(\mathrm{br} \mathrm{s}, 2 \mathrm{H}$, $\mathrm{NH}), 8.62(\mathrm{~d}, J=6.0 \mathrm{~Hz}, 4 \mathrm{H}, \operatorname{ArH}), 7.80(\mathrm{t}, J=7.7 \mathrm{~Hz}, 1 \mathrm{H}, \operatorname{ArH}), 7.56(\mathrm{~d}, J=8.0 \mathrm{~Hz}$, 2H, ArH), 7.40 (m, 4H, ArH), 7.32 (d, $J=8.2 \mathrm{~Hz}, 4 \mathrm{H}, \mathrm{ArH}), 7.30-7.23$ (m, 6H, ArH), $7.06(\mathrm{~d}, J=6.9 \mathrm{~Hz}, 4 \mathrm{H}, \mathrm{ArH}), 6.76(\mathrm{~d}, J=8.3 \mathrm{~Hz}, 4 \mathrm{H}, \mathrm{ArH}), 3.94(\mathrm{~m}, 2 \mathrm{H}, \mathrm{CHN}), 2.10$ $\left(\mathrm{s}, 3 \mathrm{H}, \mathrm{CH}_{3} \mathrm{CO}\right), 0.74 \mathrm{ppm}\left(\mathrm{d}, J=6.9 \mathrm{~Hz}, 6 \mathrm{H}, \mathrm{CH}_{3}\right) .{ }^{13} \mathrm{C} \mathrm{NMR}\left(125 \mathrm{MHz}, \mathrm{CDCl}_{3}, 25\right.$ $\left.{ }^{\circ} \mathrm{C}, \mathbf{1}^{R}(3.0 \mathrm{mM}), \mathrm{CH}_{3} \mathrm{CO}_{2} \mathrm{H}(30 \mathrm{mM})\right): \delta=176.30,162.75,149.68,142.82,141.68$, $138.95,132.43,132.26,131.68,130.92$, 129.33, 128.95, 128.28, 126.80, 125.88, 122.78, $122.51,93.50,88.31,55.75,22.37,21.32 \mathrm{ppm}$. IR $(\mathrm{KBr}): v=3429\left(v_{\mathrm{N}-\mathrm{H}}\right), 2220\left(v_{\mathrm{C} \equiv \mathrm{C}}\right)$, $1633 \mathrm{~cm}^{-1}\left(v_{\mathrm{C}=\mathrm{N}}\right)$. HRMS (FAB, NBA, $\left.[\mathrm{M}+\mathrm{H}]^{+}\right)$: calcd for $\mathrm{C}_{49} \mathrm{H}_{39} \mathrm{~N}_{4} 683.3169$, found 683.3174 .

Synthesis of $\mathbf{1}^{S}$. The title compound was prepared from $\mathbf{4}^{S}$ in the same way for $\mathbf{1}^{\boldsymbol{R}}$ and was obtained in $80 \%$ yield $(101 \mathrm{mg})$ as a pale yellow solid. Mp: $110.9-112.1{ }^{\circ} \mathrm{C}$. $[\alpha]_{\mathrm{D}}{ }^{20} 138\left(c=0.1\right.$ in $\left.\mathrm{CHCl}_{3}\right) .{ }^{1} \mathrm{H}$ NMR $\left(500 \mathrm{MHz}, \mathrm{CDCl}_{3}, 30{ }^{\circ} \mathrm{C}, \mathbf{1}^{S}(3.0 \mathrm{mM})\right.$, $\left.\mathrm{CH}_{3} \mathrm{CO}_{2} \mathrm{H}(18 \mathrm{mM})\right): \delta=13.25(\mathrm{br} \mathrm{s}, 2 \mathrm{H}, \mathrm{NH}), 8.62(\mathrm{~d}, J=6.0 \mathrm{~Hz}, 4 \mathrm{H}, \mathrm{ArH}), 7.80$ (t, $J$ $=7.8 \mathrm{~Hz}, 1 \mathrm{H}, \operatorname{ArH}), 7.56(\mathrm{~d}, J=8.0 \mathrm{~Hz}, 2 \mathrm{H}, \operatorname{ArH}), 7.40$ (m, 4H, ArH), 7.32 (d, $J=8.2$ $\mathrm{Hz}, 4 \mathrm{H}, \mathrm{ArH}), 7.30-7.23$ (m, 6H, ArH), 7.06 (d, $J=6.9 \mathrm{~Hz}, 4 \mathrm{H}, \mathrm{ArH}), 6.76$ (d, $J=8.3$ $\mathrm{Hz}, 4 \mathrm{H}, \mathrm{ArH}), 3.94$ (m, 2H, CHN), 2.10 (s, 3H, $\mathrm{CH}_{3} \mathrm{CO}$ ), $0.74 \mathrm{ppm}(\mathrm{d}, J=6.9 \mathrm{~Hz}, 6 \mathrm{H}$, $\left.\mathrm{CH}_{3}\right) .{ }^{13} \mathrm{C}$ NMR $\left(125 \mathrm{MHz}, \mathrm{CDCl}_{3}, 25{ }^{\circ} \mathrm{C}, \mathbf{1}^{\mathrm{s}}(3.0 \mathrm{mM}), \mathrm{CH}_{3} \mathrm{CO}_{2} \mathrm{H}(40 \mathrm{mM})\right): \delta=$ 
$176.65,162.78,149.51,142.72,141.67,138.94,132.44,132.29,131.80,130.92,129.34$, 128.94, 128.30, 126.79, 125.94, 122.73, 122.50, 93.60, 88.29, 55.77, 22.32, 21.22 ppm. IR (KBr): $v=3429\left(v_{\mathrm{N}-\mathrm{H}}\right), 2220\left(v_{\mathrm{C} \equiv \mathrm{C}}\right), 1632 \mathrm{~cm}^{-1}\left(v_{\mathrm{C}=\mathrm{N}}\right)$. HRMS (FAB, NBA, [M + $\mathrm{H}]^{+}$): calcd for $\mathrm{C}_{49} \mathrm{H}_{39} \mathrm{~N}_{4} 683.3169$, found 683.3181.

Synthesis of 2. A solution of compound 5 (100 mg, $0.31 \mathrm{mmol})$, 4-iodopyridine (284 mg, 4 eq.), CuI (4.4 mg, $7.5 \mathrm{~mol} \%), \operatorname{Pd}\left(\mathrm{PPh}_{3}\right)_{2} \mathrm{Cl}_{2}$ (16 mg, $\left.7.5 \mathrm{~mol} \%\right)$, and dry DMF (5 $\mathrm{mL}$ ) was degassed three times by the freeze pump and thaw (argon) method. To this solution was added dry diisopropylethylamine $(0.5 \mathrm{~mL})$ via syringe and the reaction mixture was stirred overnight at room temperature under an argon atmosphere. The solvent was evaporated to dryness. The residue was dissolved in $\mathrm{CHCl}_{3} / \mathrm{Et}_{3} \mathrm{~N}$ (100/1, $\mathrm{v} / \mathrm{v})$ and purified by flash column chromatography $\left(\mathrm{SiO}_{2}, \mathrm{CHCl}_{3} / \mathrm{MeOH}=10 / 1\right)$ to afford compound 2 in $78 \%$ yield (116 mg) as a pale yellow solid. $\mathrm{Mp}:>290{ }^{\circ} \mathrm{C}$ (dec.). ${ }^{1} \mathrm{H}$ NMR (500 MHz, DMSO- $\left.d_{6}, 25{ }^{\circ} \mathrm{C}, 3.0 \mathrm{mM}\right): \delta=13.08$ (br s, $\left.1 \mathrm{H}, \mathrm{CO}_{2} \mathrm{H}\right), 8.65(\mathrm{~m}$, 4H, ArH), 7.69 (d, $J=8.3 \mathrm{~Hz}, 4 \mathrm{H}, \operatorname{ArH}), 7.63$ (t, $J=7.7 \mathrm{~Hz}, 1 \mathrm{H}, \operatorname{ArH}), 7.55$ (d, $J=6.1$ $\mathrm{Hz}, 4 \mathrm{H}, \mathrm{ArH}), 7.52(\mathrm{~d}, J=8.3 \mathrm{~Hz}, 4 \mathrm{H}, \mathrm{ArH}), 7.47 \mathrm{ppm}(\mathrm{d}, J=7.7 \mathrm{~Hz}, 2 \mathrm{H}, \mathrm{ArH}) .{ }^{13} \mathrm{C}$ NMR $\left(125 \mathrm{MHz}, \mathrm{DMSO}-d_{6}, 25{ }^{\circ} \mathrm{C}, 3.0 \mathrm{mM}\right): \delta=169.99,150.08,141.40,138.15$, $133.76,131.87,130.23,129.55,129.34,128.95,125.53,120.57,93.36,87.46$ ppm. IR $(\mathrm{KBr}): v=3363\left(v_{\mathrm{O}-\mathrm{H}}\right), 2218\left(v_{\mathrm{C} \equiv \mathrm{C}}\right), 1708 \mathrm{~cm}^{-1}\left(v_{\mathrm{C}=\mathrm{O}}\right)$. HRMS (FAB, NBA, [M + H $\left.]^{+}\right)$: calcd for $\mathrm{C}_{33} \mathrm{H}_{21} \mathrm{~N}_{2} \mathrm{O}_{2} 477.1598$, found 477.1608.

Synthesis of Duplex $\mathbf{1}^{\boldsymbol{R}} \cdot \mathbf{2} . \quad \mathbf{1}^{\boldsymbol{R}}(0.02 \mathrm{mmol})$ and $\mathbf{2}(0.02 \mathrm{mmol})$ were suspended in $\mathrm{CHCl}_{3}(10 \mathrm{~mL})$. After stirred at room temperature, the resultant mixture became clear. The solution was filtrated through a membrane filter $(0.45 \mu \mathrm{m})$ and the filtrate was evaporated to dryness to afford the duplex $\mathbf{1}^{R} \cdot \mathbf{2}$ in $96 \%$ yield $(22.2 \mathrm{mg})$ as a white solid. Mp: $158.3-160.3{ }^{\circ} \mathrm{C} . \quad[\alpha]_{\mathrm{D}}{ }^{20}-367\left(c=0.12\right.$ in TCE). $\quad{ }^{1} \mathrm{H} \mathrm{NMR}\left(500 \mathrm{MHz}, \mathrm{CDCl}_{3}, 30\right.$ $\left.{ }^{\circ} \mathrm{C}, 1.0 \mathrm{mM}\right): \delta=13.48(\mathrm{~d}, J=9.1 \mathrm{~Hz}, 2 \mathrm{H}, \mathrm{NH}), 8.51(\mathrm{~m}, 8 \mathrm{H}, \mathrm{ArH}), 7.80(\mathrm{~m}, 4 \mathrm{H}, \mathrm{ArH})$, $7.77(\mathrm{t}, J=7.7 \mathrm{~Hz}, 1 \mathrm{H}, \mathrm{ArH}), 7.70(\mathrm{~d}, J=8.0 \mathrm{~Hz}, 4 \mathrm{H}, \mathrm{ArH}), 7.53(\mathrm{~d}, J=8.0 \mathrm{~Hz}, 2 \mathrm{H}$, $\operatorname{ArH}), 7.50$ (d, $J=8.3 \mathrm{~Hz}, 4 \mathrm{H}, \operatorname{ArH}), 7.44-7.38$ (m, 3H, ArH), 7.34 (d, $J=6.1 \mathrm{~Hz}, 4 \mathrm{H}$, ArH), 7.30 (d, $J=5.8 \mathrm{~Hz}, 4 \mathrm{H}, \mathrm{ArH}), 7.13$ (t, $J=7.4 \mathrm{~Hz}, 4 \mathrm{H}, \mathrm{ArH}), 7.06$ (t, $J=7.2 \mathrm{~Hz}$, 2H, ArH), 6.78 (d, $J=7.4 \mathrm{~Hz}, 4 \mathrm{H}, \mathrm{ArH}), 6.70$ (d, $J=8.3 \mathrm{~Hz}, 4 \mathrm{H}, \mathrm{ArH}), 3.80$ (m, 2H, $\mathrm{CHN}), 0.65 \mathrm{ppm}\left(\mathrm{d}, J=6.9 \mathrm{~Hz}, 6 \mathrm{H}, \mathrm{CH}_{3}\right) .{ }^{13} \mathrm{C} \mathrm{NMR}\left(125 \mathrm{MHz}, \mathrm{CDCl}_{3}, 25{ }^{\circ} \mathrm{C}, 1.0\right.$ $\mathrm{mM}): \delta=162.41,150.01,143.71,142.59,141.26,139.04,132.24,132.12,131.71$, 
131.52, 131.04, 130.43, 129.60, 129.31, 129.16, 128.96, 127.87, 127.29, 126.67, 125.61, $125.53,123.18,122.49,120.54,94.82,93.07,88.82,87.63,55.78,22.67$ ppm (31 peaks for 34C). IR (KBr): $v=3420\left(v_{\mathrm{N}-\mathrm{H}}\right), 2219\left(v_{\mathrm{C}=\mathrm{C}}\right), 1653\left(v_{\mathrm{C}=\mathrm{O}}\right), 1590 \mathrm{~cm}^{-1}\left(v_{\mathrm{C}=\mathrm{N}}\right)$. CSIMS: $m / z, 1159.55\left([\mathrm{M}+\mathrm{H}]^{+}\right)$. Anal. Calcd for $\mathrm{C}_{82} \mathrm{H}_{58} \mathrm{~N}_{6} \mathrm{O}_{2}$ : Anal. Calcd for $\mathrm{C}_{82} \mathrm{H}_{58} \mathrm{~N}_{6} \mathrm{O}_{2}$ : C, 84.95; H, 5.04; N, 7.25. Found: C, 84.95; H, 4.86; N, 7.09.

Synthesis of Duplex $\mathbf{1}^{S} \cdot \mathbf{2}$. The title compound was prepared from $\mathbf{1}^{S}$ and $\mathbf{2}$ in the same way for $\mathbf{1}^{\boldsymbol{R}} \cdot \mathbf{2}$, and was obtained in $99 \%$ yield $(23.0 \mathrm{mg})$ as a white solid. Mp: 158.3-160.1 ${ }^{\circ} \mathrm{C} . \quad[\alpha]_{\mathrm{D}}{ }^{20}+369\left(c=0.12\right.$ in TCE). $\quad{ }^{1} \mathrm{H}$ NMR $\left(500 \mathrm{MHz}, \mathrm{CDCl}_{3}, 30{ }^{\circ} \mathrm{C}\right.$, $1.0 \mathrm{mM}): \delta=13.48(\mathrm{~d}, J=9.1 \mathrm{~Hz}, 2 \mathrm{H}, \mathrm{NH}), 8.51(\mathrm{~m}, 8 \mathrm{H}, \mathrm{ArH}), 7.80(\mathrm{~m}, 4 \mathrm{H}, \operatorname{ArH})$, $7.77(\mathrm{t}, J=7.7 \mathrm{~Hz}, 1 \mathrm{H}, \mathrm{ArH}), 7.70(\mathrm{~d}, J=8.0 \mathrm{~Hz}, 4 \mathrm{H}, \mathrm{ArH}), 7.53(\mathrm{~d}, J=8.0 \mathrm{~Hz}, 2 \mathrm{H}$, ArH), 7.49 (d, $J=8.3 \mathrm{~Hz}, 4 \mathrm{H}, \operatorname{ArH}), 7.44-7.38$ (m, 3H, ArH), 7.34 (d, $J=6.1 \mathrm{~Hz}, 4 \mathrm{H}$, ArH), 7.30 (d, $J=5.8 \mathrm{~Hz}, 4 \mathrm{H}, \mathrm{ArH}), 7.13$ (t, $J=7.4 \mathrm{~Hz}, 4 \mathrm{H}, \mathrm{ArH}), 7.06$ (t, $J=7.2 \mathrm{~Hz}$, 2H, ArH), 6.78 (d, $J=7.5 \mathrm{~Hz}, 4 \mathrm{H}, \operatorname{ArH}), 6.70(\mathrm{~d}, J=8.3 \mathrm{~Hz}, 4 \mathrm{H}, \operatorname{ArH}), 3.80$ (m, 2H, $\mathrm{CHN}), 0.65 \mathrm{ppm}\left(\mathrm{d}, J=6.9 \mathrm{~Hz}, 6 \mathrm{H}, \mathrm{CH}_{3}\right) .{ }^{13} \mathrm{C} \mathrm{NMR}\left(125 \mathrm{MHz}, \mathrm{CDCl}_{3}, 25{ }^{\circ} \mathrm{C}, 1.0\right.$ $\mathrm{mM}): \delta=162.40,150.02,143.74,142.60,141.27,139.05,132.24,132.10,131.69$, 131.53, 131.04, 130.43, 129.59, 129.31, 129.16, 128.98, 127.87, 127.20, 126.67, 125.61, $125.53,123.18,122.50,120.55,94.78,93.06,88.83,87.63,55.78,22.67$ ppm (31 peaks for 34C). IR (KBr): $v=3425\left(v_{\mathrm{N}-\mathrm{H}}\right), 2219\left(v_{\mathrm{C} \equiv \mathrm{C}}\right), 1652\left(v_{\mathrm{C}=\mathrm{O}}\right), 1591 \mathrm{~cm}^{-1}\left(v_{\mathrm{C}=\mathrm{N}}\right)$. CSIMS: $m / z$ 1159.55 $\left([\mathrm{M}+\mathrm{H}]^{+}\right)$. Anal. Calcd for $\mathrm{C}_{82} \mathrm{H}_{58} \mathrm{~N}_{6} \mathrm{O}_{2}: \mathrm{C}, 84.95 ; \mathrm{H}, 5.04 ; \mathrm{N}, 7.25$. Found: C, 84.70; H, 4.98; N, 7.15.

Representative Procedure for Supramolecular Polymerization. A solution of $\mathbf{1}^{\boldsymbol{R}} \cdot \mathbf{2}$ in TCE $(2.0 \mathrm{mM}, \quad 1.0 \mathrm{~mL})$ and a solution of cis-diphenylbis(dimethyl sulfoxide)platinum(II) $)^{2}\left(\right.$ cis $\left.-\mathrm{PtPh}_{2}(\mathrm{dmso})_{2}\right)$ in TCE $(4.0 \mathrm{mM}, 1.0 \mathrm{~mL})$ were mixed at ambient temperature, and the resulting solution was subjected to spectroscopic analyses. $\mathbf{3}^{R} . \quad[\alpha]_{\mathrm{D}}^{20}-209(c=0.19$ in TCE $) . \quad 3^{S} . \quad[\alpha]_{\mathrm{D}}^{20}+214(c=0.19$ in TCE $)$. 
4. Absorption and CD Spectra of the Duplexes $1^{R} \cdot 2$ and $1^{S} \cdot 2$.
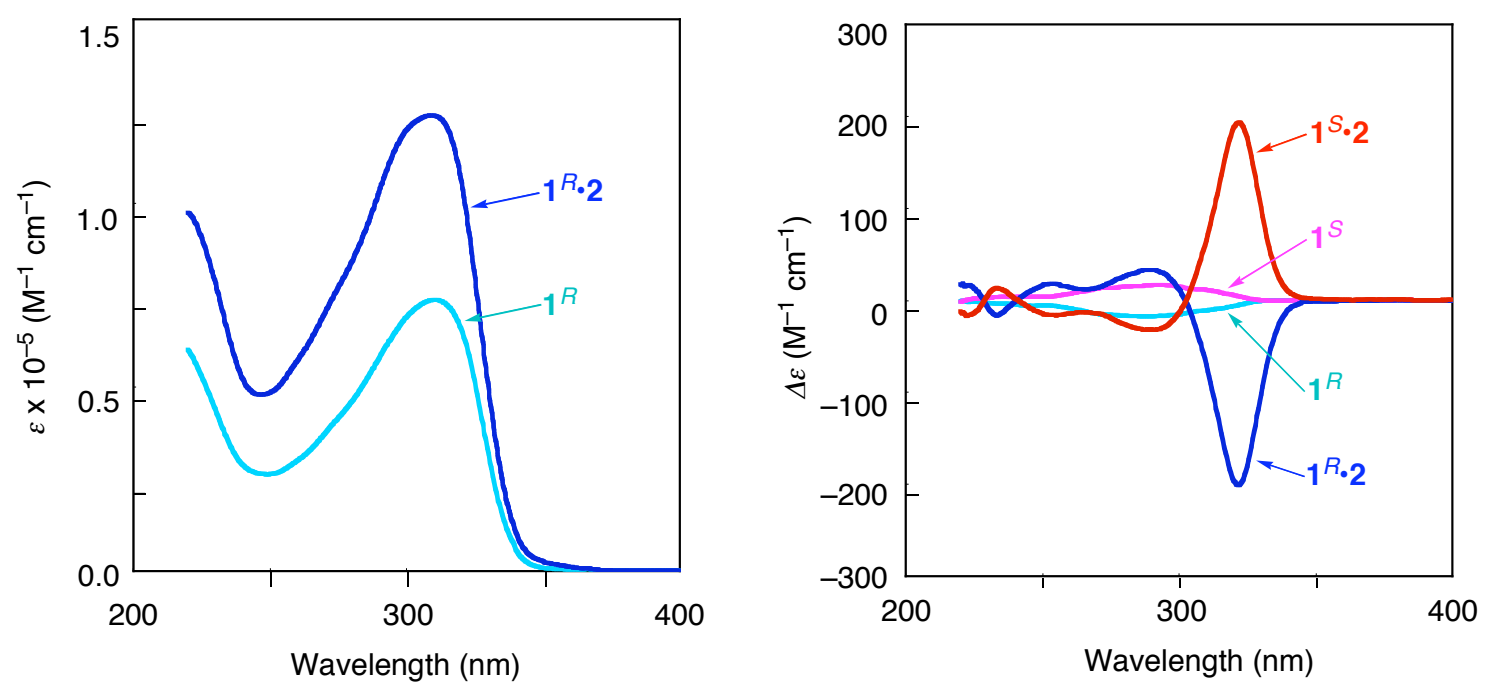

Figure S1. Absorption and CD spectra $(1.0 \mathrm{mM}, 0.1 \mathrm{~mm}$ cell $)$ of $\mathbf{1}^{R}, \mathbf{1}^{S}, \mathbf{1}^{R} \cdot \mathbf{2}$, and $\mathbf{1}^{S} \cdot \mathbf{2}$ in $\mathrm{CHCl}_{3}$ at $\mathrm{ca} .25^{\circ} \mathrm{C}$.

5. ${ }^{1} \mathrm{H}$ NMR (500 MHz, $\left.25{ }^{\circ} \mathrm{C}\right)$ Spectra of $1^{R}, 2$, and $1^{R} \cdot 2$.

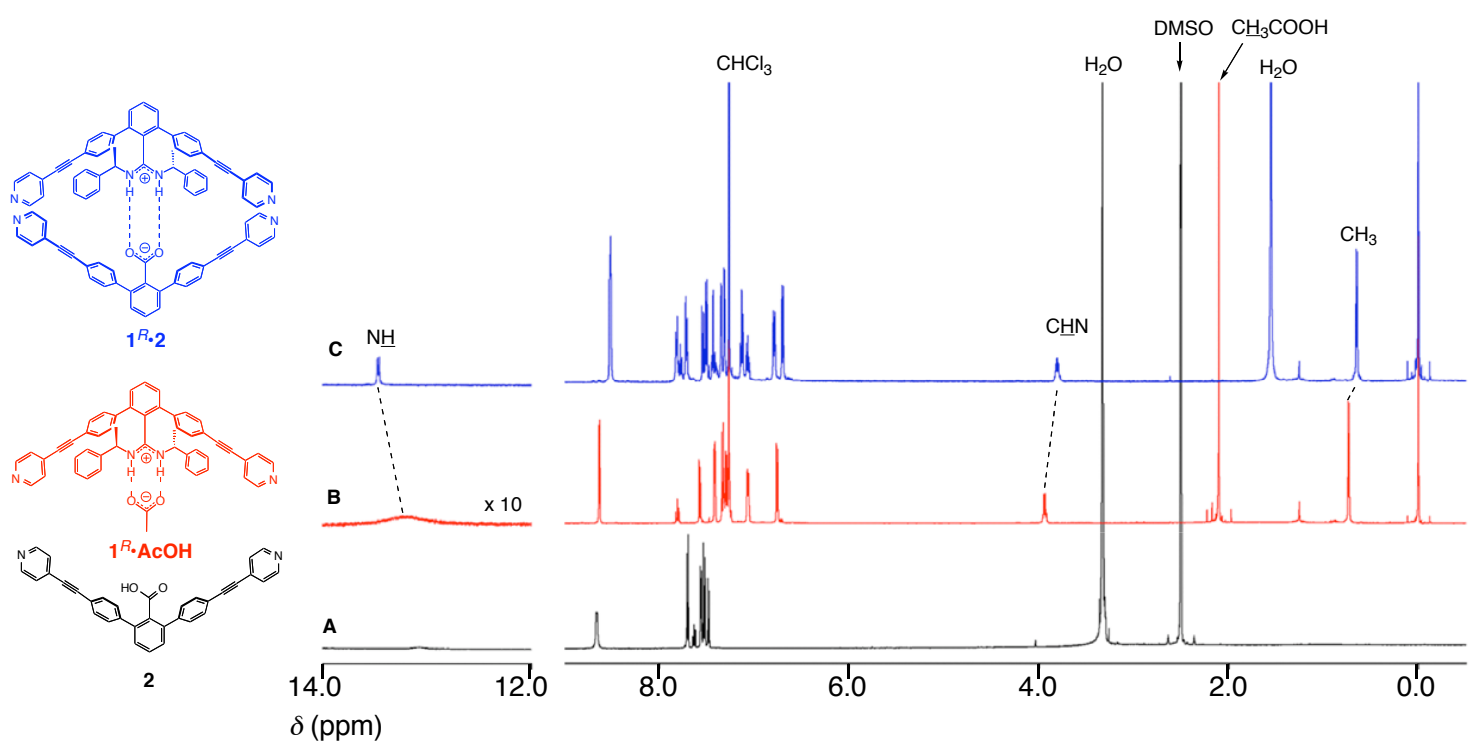

Figure S2. ${ }^{1} \mathrm{H}$ NMR spectra of (A) $2(3.0 \mathrm{mM})$ in DMSO- $d_{6},(\mathrm{~B}) \mathbf{1}^{\boldsymbol{R}} \cdot \mathrm{AcOH}\left(\mathbf{1}^{\boldsymbol{R}}(3.0\right.$ $\mathrm{mM})$ and $\mathrm{AcOH}(30 \mathrm{mM}))$ in $\mathrm{CDCl}_{3}$, and $(\mathrm{C}) \mathbf{1}^{R} \cdot \mathbf{2}(1.0 \mathrm{mM})$ in $\mathrm{CDCl}_{3}$ at $25{ }^{\circ} \mathrm{C}$. 
6. CSI Mass Spectra of the Duplexes $1^{R} \cdot 2$ and $1^{S} \cdot 2$.

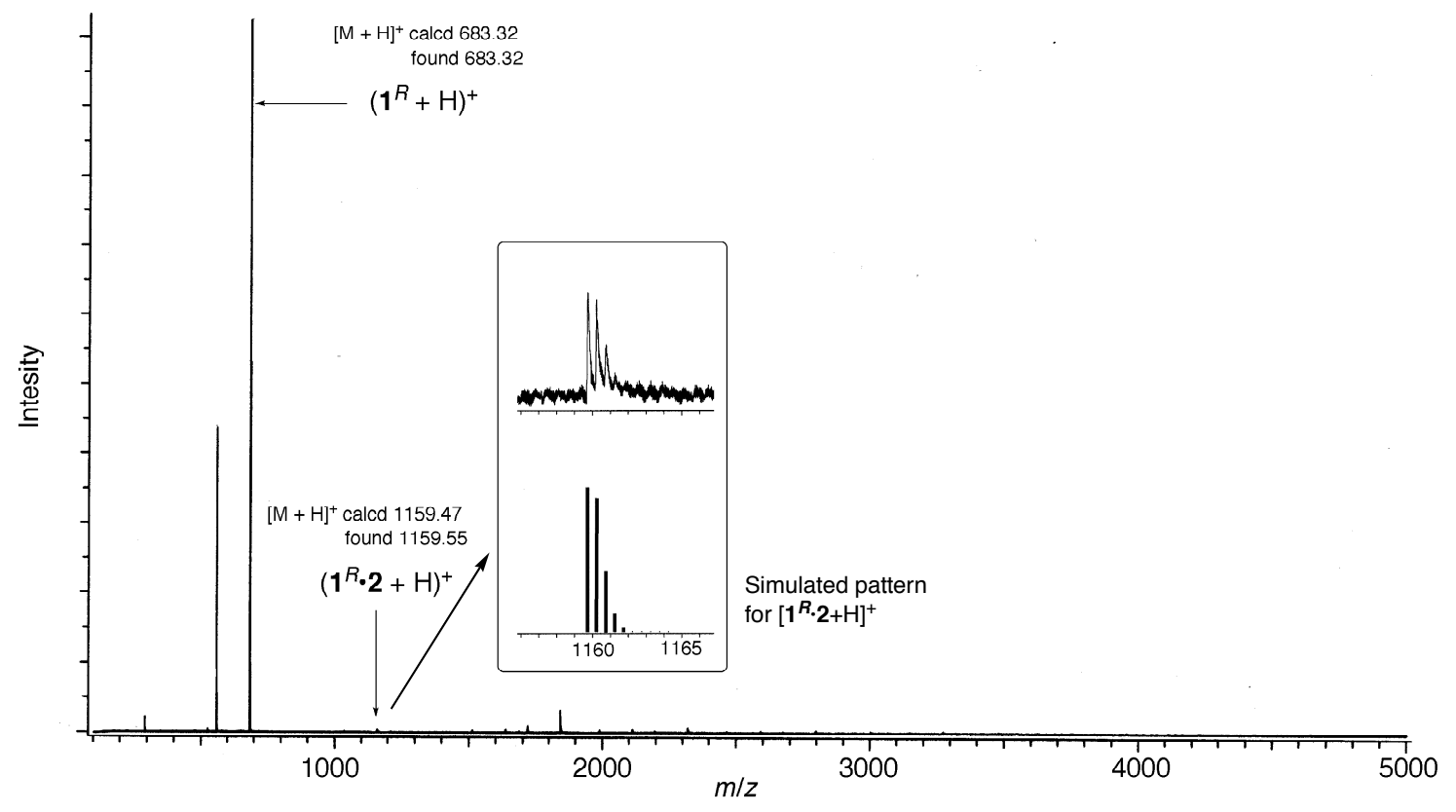

Figure S2. Positive-mode CSI MS spectrum of $\mathbf{1}^{\boldsymbol{R}} \cdot \mathbf{2}\left(1.0 \mathrm{mM}\right.$ in $\mathrm{CHCl}_{3}$, ion source temperature $=-15^{\circ} \mathrm{C}$ ).

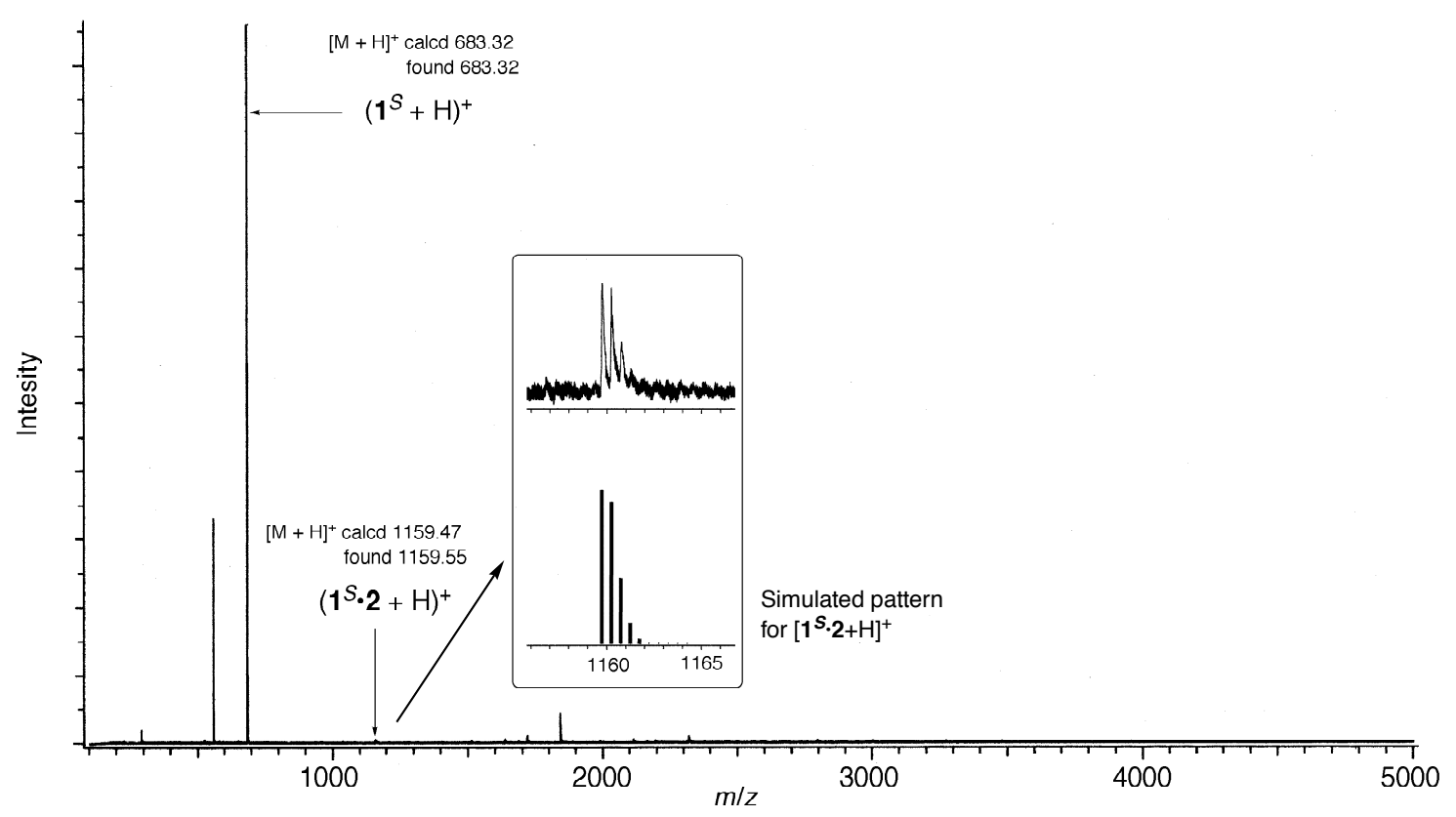

Figure S4. Positive-mode CSI MS spectrum of $\mathbf{1}^{S} \cdot \mathbf{2} \quad\left(1.0 \mathrm{mM}\right.$ in $\mathrm{CHCl}_{3}$, ion source temperature $=-15^{\circ} \mathrm{C}$ ). 


\section{DOSY NMR Spectra of $1^{R} \cdot 2$ and $3^{R}$.}

A

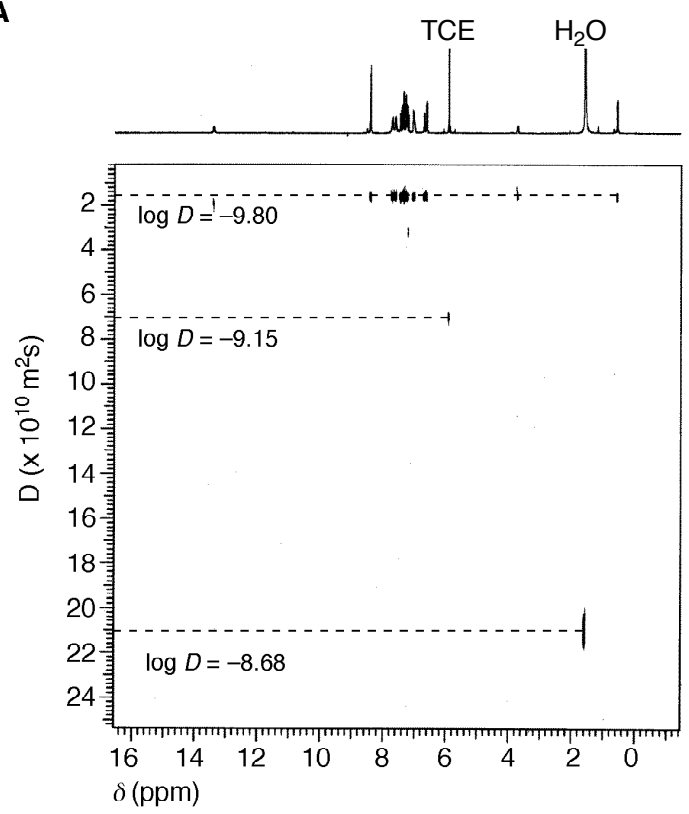

B

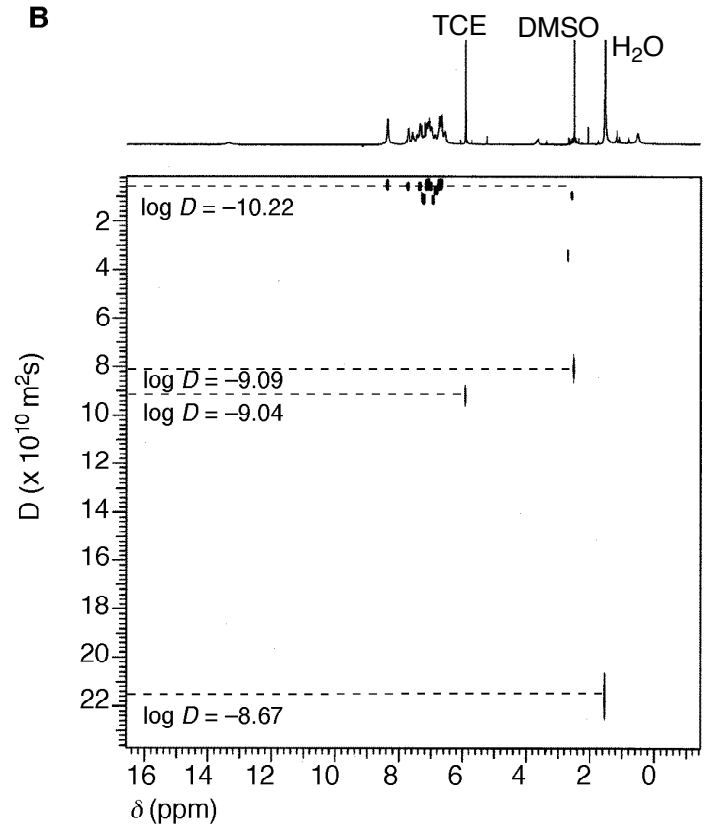

Figure S5. DOSY spectra of (A) $\mathbf{1}^{\boldsymbol{R}} \cdot \mathbf{2}$ in TCE- $d_{2}(1.0 \mathrm{mM})$ and (B) $\mathbf{3}^{\boldsymbol{R}}$ in TCE- $d_{2}(1.0$ $\mathrm{mM}$ per repeating unit) at $25^{\circ} \mathrm{C}$.

8. DLS Study of the Double Helical Coordination Polymer $3^{R}$.

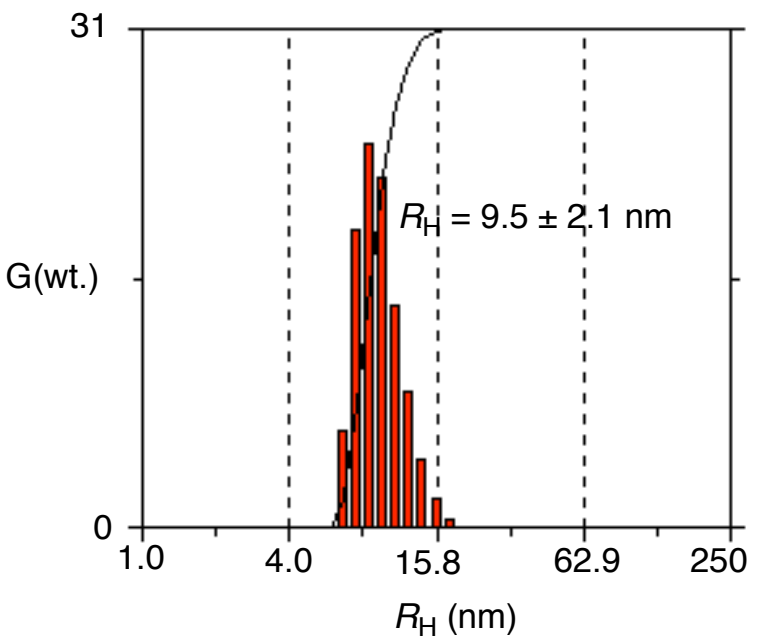

Figure S6. Histogram analysis of DLS measurement of $\mathbf{3}^{R}$ in TCE (1.0 mM per repeating unit) at $c a .25^{\circ} \mathrm{C}$. 


\section{Effect of Feed Ratio.}
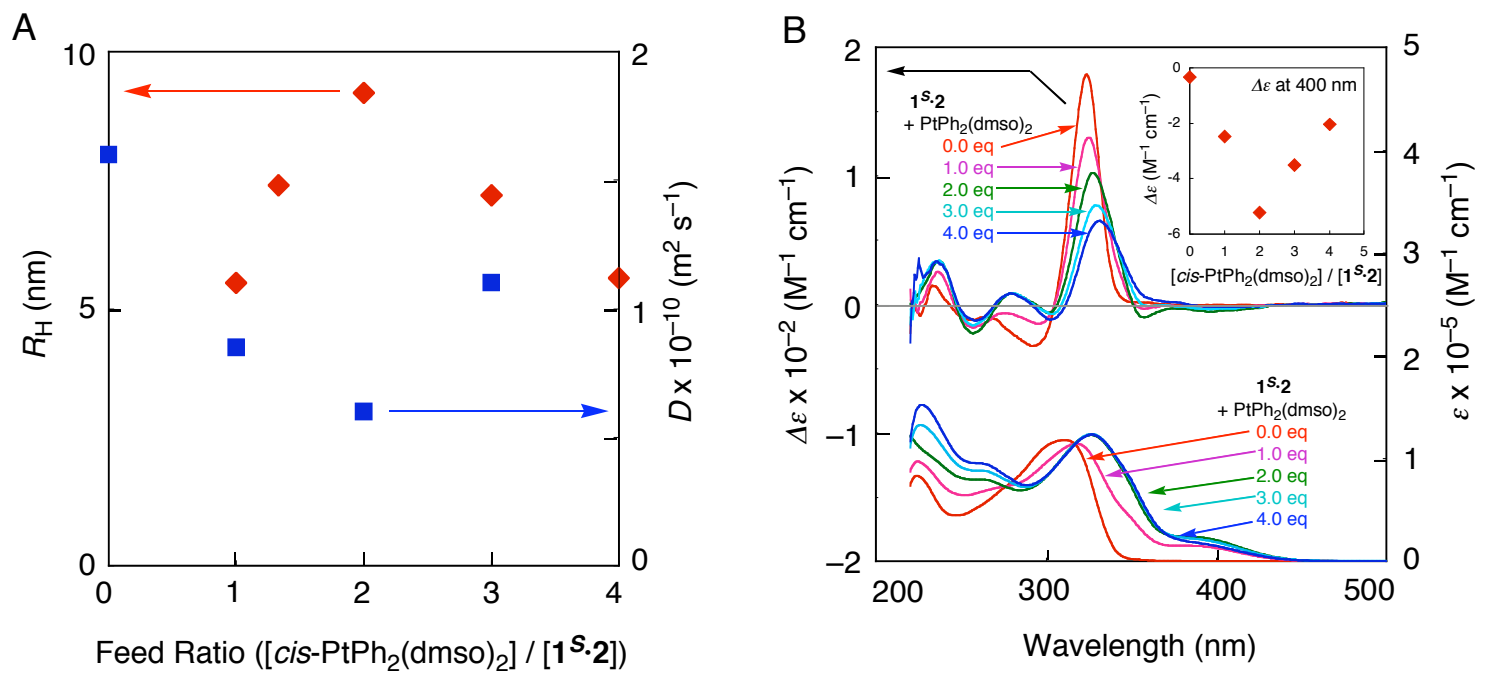

Figure S7. (A) Changes in the average hydrodynamic radius $\left(R_{\mathrm{H}}\right.$, red $)$ determined by DLS measurements and the diffusion constant ( $D$, blue) measured by DOSY experiments of $\mathbf{3}^{S}$ prepared at different feed ratios in TCE $\left(\left[\mathbf{1}^{S} \cdot \mathbf{2}\right]=1.0 \mathrm{mM}\right)$. (B) CD spectral changes of $\mathbf{3}^{S}$ prepared at different feed ratios in TCE $\left(\left[\mathbf{1}^{S} \cdot \mathbf{2}\right]=1.0 \mathrm{mM}\right)$.

\section{Effect of AcOH on the CD Spectra and Hydrodynamic Dimension of $3^{S}$.}
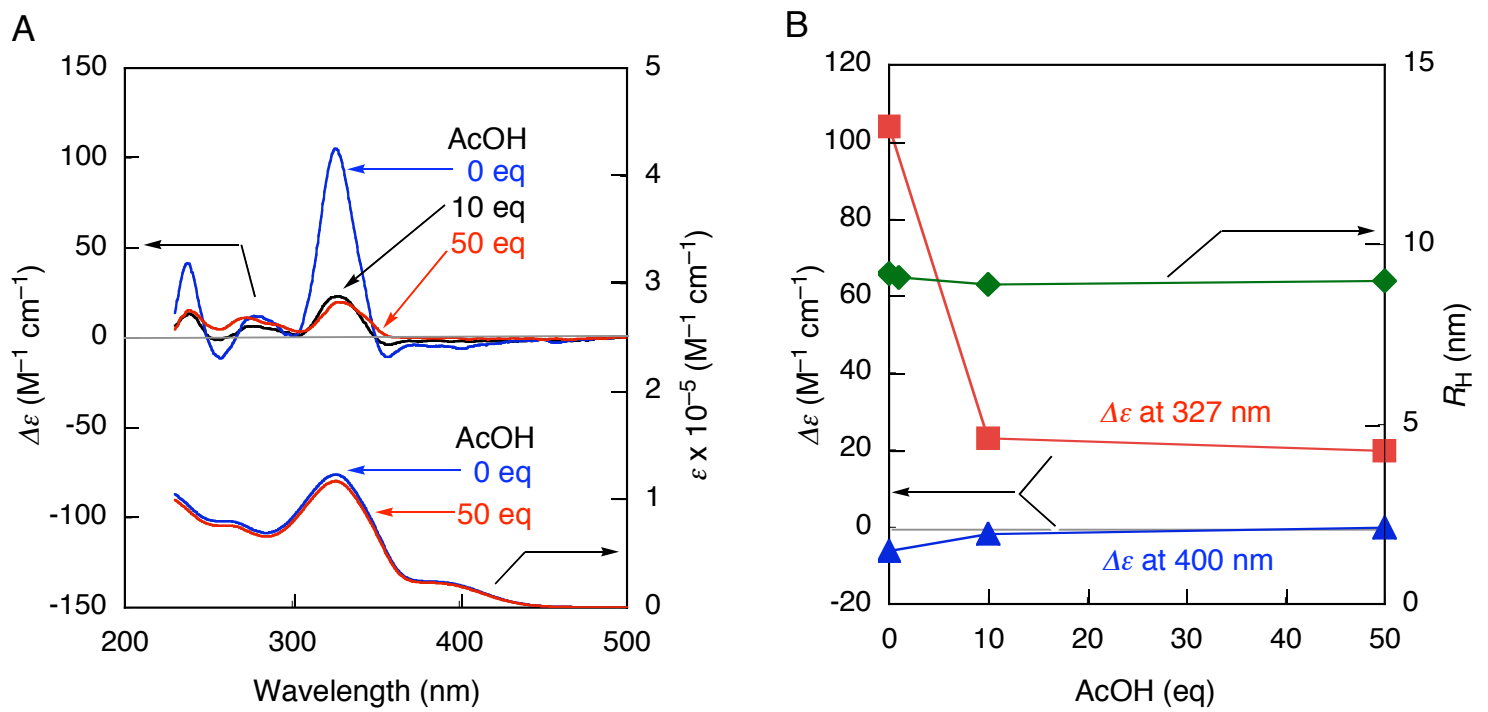

Figure S8. (A) CD and absorption spectral changes of $\mathbf{3}^{S}$ in TCE (1.0 mM per repeating unit) in the presence of increasing amounts of $\mathrm{AcOH}$ (0 (blue), 10 (black), and $50 \mathrm{eq}$ (red)). (B) Plots of $\Delta \varepsilon$ at 327 (red) and $400 \mathrm{~nm}$ (blue), and the $R_{\mathrm{H}}$ value (green) of $\mathbf{3}^{S}$ versus the amount of $\mathrm{AcOH}$ added. 
11. Molecular Mechanics Calculation of a Trimer Model of $3^{R}$. The MMcalculation of a trimer model of $\mathbf{3}^{R}$ was performed on a Windows XP PC with the Materials Studio package (Version 3.0; Accelrys Inc.). The initial structure of the trimer model was constructed using the crystal structure of a double helix comprising a $m$-terphenyl-bound chiral amidine and a $m$-terphenyl-bound achiral carboxylic acid. ${ }^{1}$ The geometry optimization of the trimer model was carried out by the steepest descent, conjugated gradient and finally Quasi-Newton methods (smart algorism) with the Forcite program using the Universal force field. The energy minimization was continued until the root-mean-square (rms) value became less than $0.1 \mathrm{kcal} \mathrm{mol}^{-1} \AA^{-1}$.

Top view

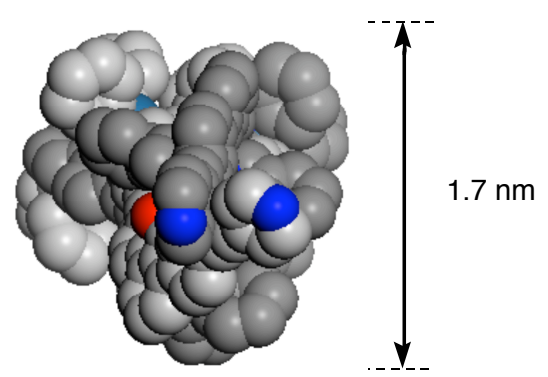

Side view

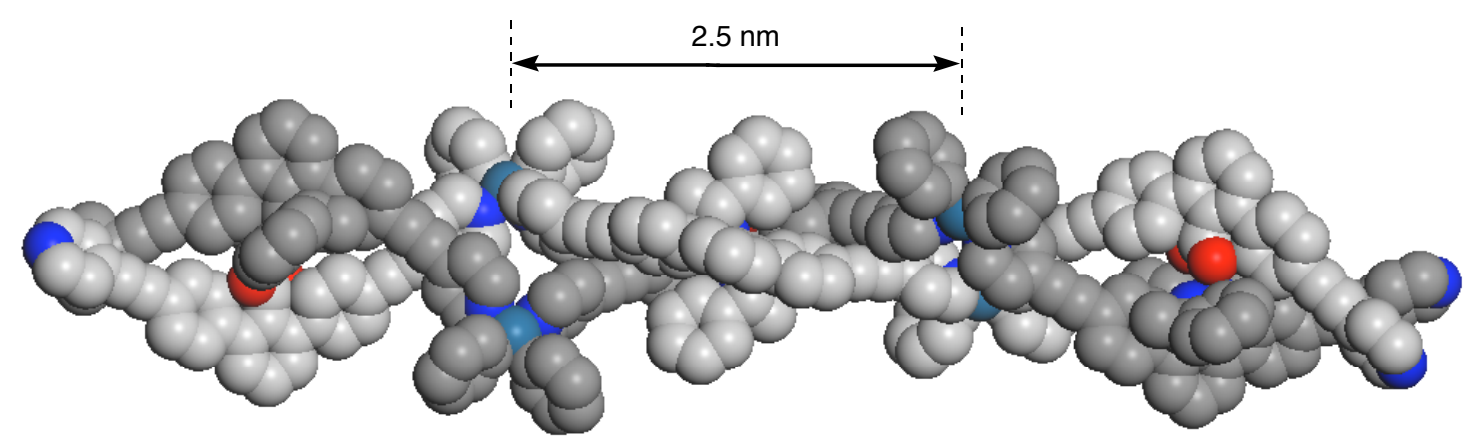

Figure S9. A space-filling drawing of a trimer model of $\mathbf{3}^{R}$. Hydrogen atoms are omitted for clarify. 
12. AFM Measurements. The polymer samples for the AFM measurements were prepared by casting $10 \mu \mathrm{L}$ aliquots of a solution of $3^{R}$ or $\mathbf{1}^{R} \cdot \mathbf{2}$ in TCE $(2 \mu \mathrm{M})$ on HOPG. The HOPG substrate was stored at $c a .25{ }^{\circ} \mathrm{C}$ in a clean bench for $10 \mathrm{~min}$ and was subsequently dried under vacuum for $2 \mathrm{~h}$. The AFM measurements were performed using a Nanoscope IIIa microscope (Veeco Instruments, Santa Barbara, CA) at $c a .25^{\circ} \mathrm{C}$ with standard silicon cantilevers ( $\mathrm{NCH}$, NanoWorld, Neuchâtel, Switzerland) in the tapping mode. The Nanoscope image processing software was used for the image analysis.

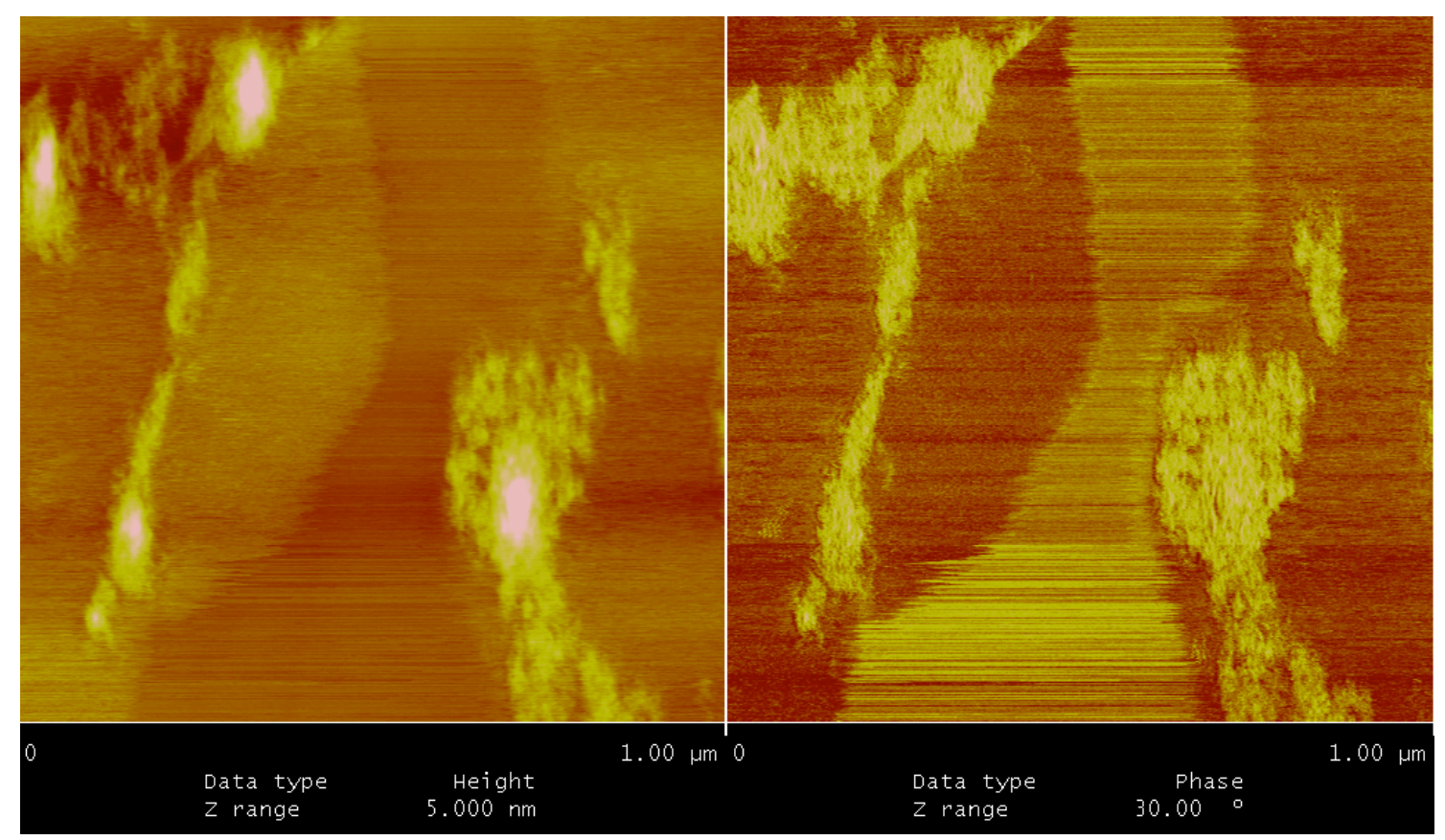

Figure S10. AFM height (left) and phase (right) images of $\mathbf{1}^{\boldsymbol{R}} \cdot \mathbf{2}$ on HOPG.

\section{References.}

(1) Tanaka, Y.; Katagiri, H.; Furusho, Y.; Yashima, E. Angew. Chem., Int. Ed. 2005, 44, 3867-3870.

(2) Lanza, S.; Minniti, D.; Moore, P.; Sachinidis, J.; Romeo, R.; Tobe, M. L. Inorg. Chem. 1984, 23, 4428-4433. 